Tibor Kálmán*, Xi Kong und Ulrich Schwardmann

\title{
Die digitale Forschungsinfrastruktur DARIAH-DE: Angebotspalette für die Geistes- und Kulturwissenschaften
}

DOI 10.1515/bfp-2016-0041

Zusammenfassung: DARIAH-DE stellt ein breites Spektrum von Diensten und Tools einer digitalen Forschungsinfrastruktur, zugeschnitten auf digital arbeitende Geistesund Kulturwissenschaftler, bereit. Die angebotenen Dienste setzen auf robuste, modulare, nachhaltige und hochverfügbare technische Basiskomponenten aktueller Technologien, und weisen eine hohe Flexibilität für zukünftige erweiterte Angebote auf.

Schlüsselwörter: DARIAH-DE; Digitale Forschungsinfrastruktur; Basiskomponenten

The Digital Research Infrastructure DARIAH-DE: Services for the Arts and Humanities

Abstract: DARIAH-DE provides a broad spectrum of services and tools of a digital research infrastructure tailored for digitally working researchers from the Arts and Humanities. The services rely on robust, modular, sustainable, and high-available technical components and present a high flexibility for future extensions.

Keywords: DARIAH-DE; digital research infrastructure; basis components

Inhalt

1 Die Angebotspalette einer Forschungsinfrastruktur . . . . . . . . . . . . . . . . . . . 234

1.1 Service Packages für die Digital Humanities . . . 234

2 Die Beschreibung der Angebote am Beispiel von DARIAH-DE ................... 235

2.1 Services Technische Infrastruktur und Unterstützungen . . . . . . . . . . . . . . . 236

2.2 Services Basisdienste . . . . . . . . . . . . 237

2.3 Services Generische Dienste . . . . . . . . . . 239

2.4 Service Operative IT-Dienste . . . . . . . . . 240

*Kontaktperson: Tibor Kálmán, tkalman@gwdg.de Xi Kong, xi.kong@gwdg.de Ulrich Schwardmann, uschwar1@gwdg.de
3 Einbindung fachspezifischer Infrastrukturkomponenten . . . . . . . . . . . . . . . . . . 242

4 Innovationszyklen und Nachhaltigkeit der Angebote am Beispiel von DARIAH-DE . . . . . . . . . . 242

5 Zusammenfassung ............... 243

\section{Die Angebotspalette einer Forschungsinfrastruktur}

Wer digital forscht und Methoden und Verfahren der Digital Humanities anwendet, braucht entsprechende Tools und Dienste. DARIAH-DE stellt den Geistes- und Kulturwissenschaftlern eine Reihe von Werkzeugen zur Verfügung und hält diese nachhaltig zur Nutzung bereit (siehe Abb. 1).

\subsection{Service Packages für die Digital Humanities}

Aktuell werden drei verschiedene Service Packages für die Digital Humanities, Bronze, Silber und Gold, konzipiert. Diese sollen für verschieden große Projekte und Institutionen interessante Bündelungen von Angeboten des DARIAH-DE bieten, welche als Teil des Nachhaltigkeitskonzepts $\mathrm{zu}$ sehen sind. Aktuell sind folgende Konfigurationen geplant:

Tab. 1: Service Packages für die Digital Humanites

\begin{tabular}{llll}
\hline & Bronze & Silber & Gold \\
\hline Support & $\begin{array}{l}\text { Nutzerforen, } \\
\text { FAQs, Tutorials }\end{array}$ & $\begin{array}{l}\text { Bronze }+ \\
\text { Helpdesk }\end{array}$ & $\begin{array}{l}\text { Bronze }+ \\
\text { Helpdesk }\end{array}$ \\
\hline $\begin{array}{l}\text { Fachwissen- } \\
\text { schaftliche } \\
\text { Beratung }\end{array}$ & 4 Std. (remote) & 8 Std. (remote) & $\begin{array}{l}16 \text { Std. } \\
\text { (remote) }\end{array}$ \\
\hline Etherpad & + & & \\
\hline $\begin{array}{l}\text { Confluence } \\
\text { inkl. AAl }\end{array}$ & 5 Spaces,20 & 30 Spaces,200 & $\begin{array}{l}100 \text { Spaces, } \\
\text { Nutzer }\end{array}$ \\
\hline
\end{tabular}




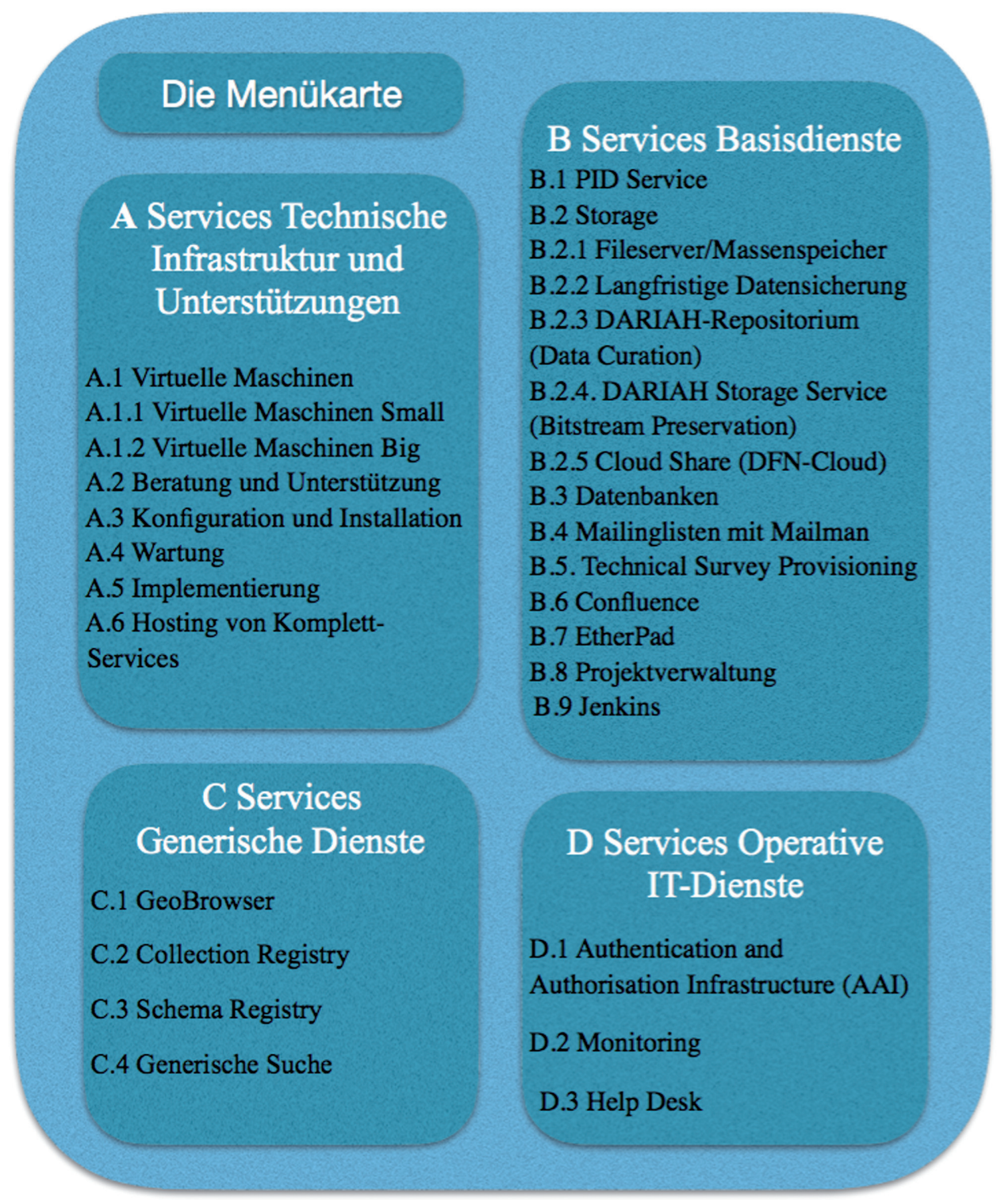

Abb. 1: DARIAH Service Menükarte

\begin{tabular}{llll}
\hline & Bronze & Silber & Gold \\
\hline $\begin{array}{l}\text { Storage } \\
\text { (Cloud Share) }\end{array}$ & 1 TB & 2 TB & $10 \mathrm{~TB}$ \\
\hline Repository & $5 \mathrm{~GB}$ & $1 \mathrm{~TB}$ & $10 \mathrm{~TB}$ \\
\hline $\begin{array}{l}\text { Langzeitarchi- } \\
\text { vierung }\end{array}$ & + & + & + \\
\hline VMs & - & + & + \\
\hline $\begin{array}{l}\text { Developer } \\
\text { Portal (SVN } /\end{array}$ & - & $5 \mathrm{~GB}$ & $50 \mathrm{~GB}$ \\
$\begin{array}{l}\text { GIT Space) } \\
\text { PID-Service }\end{array}$ & & \\
\hline Monitoring & - & 50.000 PIDs & 1.000 .000 \\
\hline
\end{tabular}

\section{Die Beschreibung der Angebote am Beispiel von DARIAH-DE}

Die wichtigsten Anforderungen an die digitale Infrastruktur, die von DARIAH-DE den Wissenschaftlern in den Digital Humanities bereitgestellt wird, sind wohl eine angemessene Vielfalt der Dienste, eine einfache Nutzbarkeit und leichte Verständlichkeit des Angebotes und eine hohe Verfügbarkeit und Performanz der angebotenen Services. Eine Umsetzung dieser Anforderungen durch die technische Infrastruktur sowie ihre stete Anpassung an die Bedürfnisse der Wissenschaftler setzt ein modulares Vorgehen voraus, was auf technischer Ebene auf robusten und hoch verfügbaren Komponenten hoch aktueller Technologie aufsetzt und auf organisatorischer Ebene die Prozesse des Technology-Watch und der Einbindens neuer 
DARIAH-DE: DIGITALE FORSCHUNGSINFRASTRUKTUR FÜR DIE GEISTES- UND KULTURWISSENSCHAFTEN

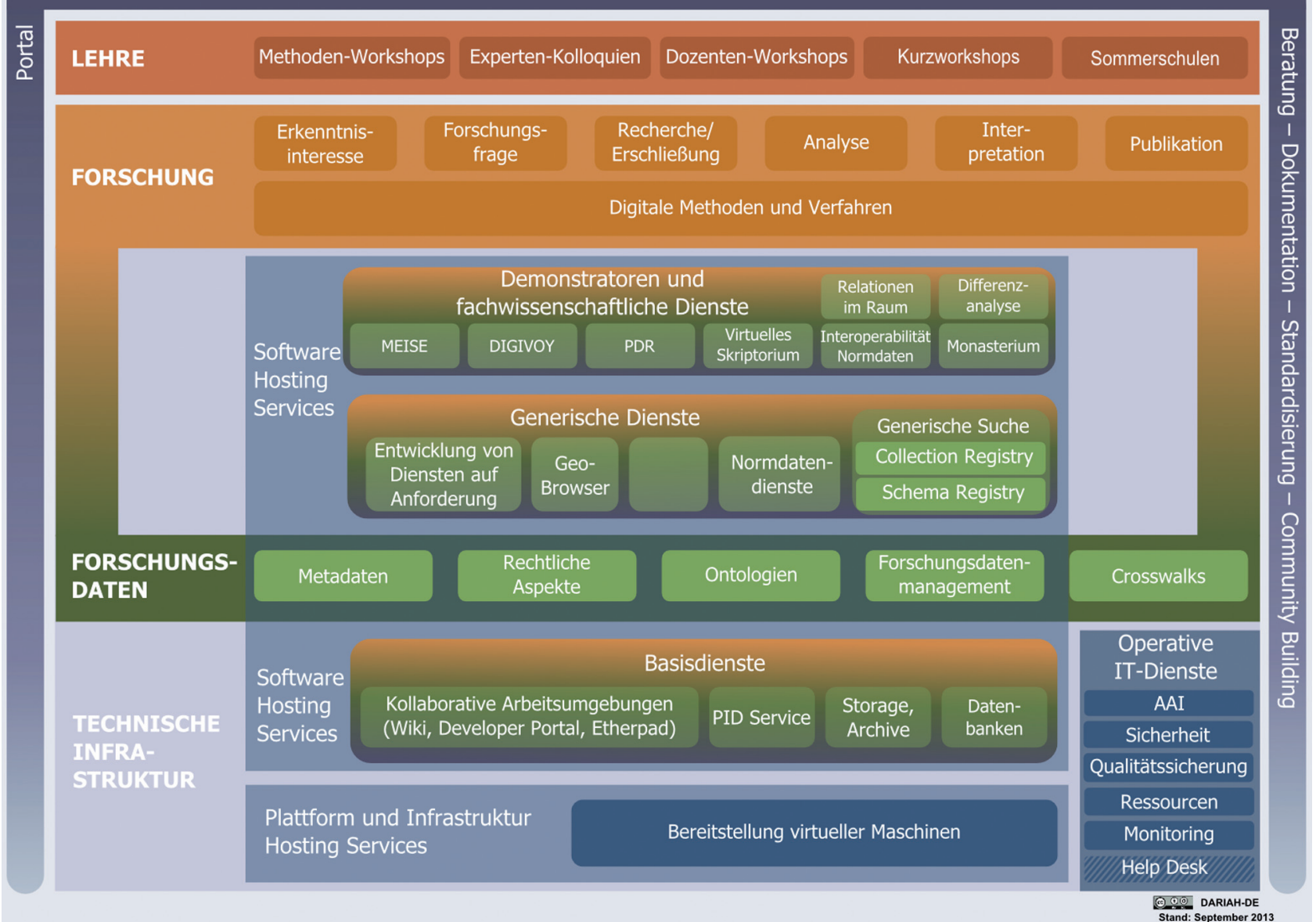

Abb. 2: DARIAH-DE: Digitale Forschungsinfrastruktur für die Geistes- und Kulturwissenschaften ${ }^{1}$

Services auf diese Module abbildet. Die Angebotspalette in DARIAH-DE stellt dabei die aktuell verfügbaren Dienste in DARIAH-DE dar und ist sowohl eine Darstellung der technischen Komponenten in DARIAH-DE, als auch ihrer Modularität. Sie gibt ebenso beispielhaft einen Überblick, welche höheren Dienste sich auf den Komponenten aufbauen lassen, und damit auch einen Hinweis, wie zukünftige Dienste zusammengestellt werden können. Den Wissenschaftlern können so entsprechend der DARIAH-DEForschungsinfrastrukturgrafik (siehe Abb. 2) Angebote gemacht werden, die ihre Forschung unterstützen und modernen technischen Ansprüchen genügen.

1 https://wiki.de.dariah.eu/download/attachments/30376358/14061 2_dariah-3.7_de.png?version=1\&modificationDate=1403868459862\& api=v2.

\subsection{Services Technische Infrastruktur und Unterstützungen}

Es werden virtuelle Maschinen, VMs, in denen sich Software-Programme oder Betriebssysteme ausführen und installieren lassen, bereitgestellt. Sie ermöglichen den Aufbau komplexer Services und Workflows und sind somit unverzichtbare Bestandteile der digitalen Forschungsinfrastruktur (A1 Virtuelle Maschinen, vgl. Abb. 3).

\subsubsection{Virtuelle Maschine Small und Virtuelle Maschine Big}

Bei der Bereitstellung von VMs legt DARIAH-DE besonderen Wert auf die Bedürfnisse der Geistes- und Kulturwissenschaftler. Aktuell werden zwei Kategorien Virtuelle Maschine Small, die derzeit 2 virtuelle CPUs, 2 GB RAM und 200 GB HDD und Virtuelle Maschine Big, die derzeit 4 virtuelle CPUs, 16 GB RAM und 1TB Massenspeicher hat, 


\section{Cloud Server}

Our Private Cloud for your dynamic, safe and scalable computing power

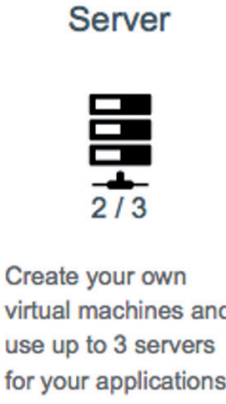

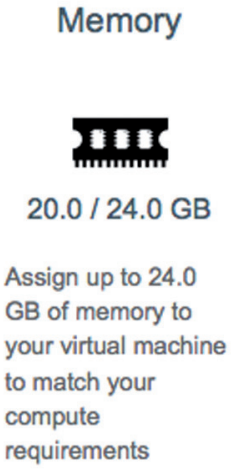

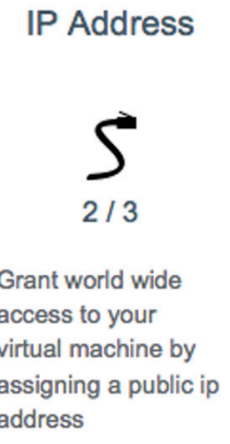

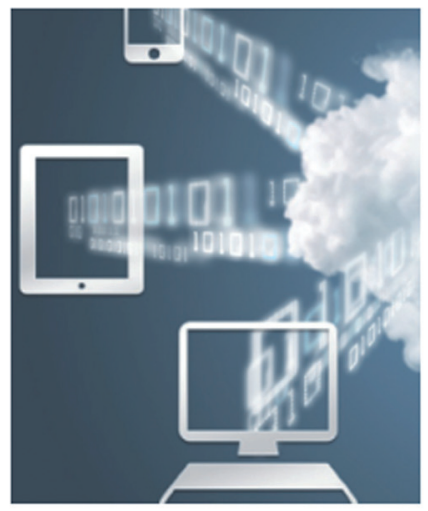

Abb. 3: Virtuelle Maschinen

angeboten. Das nachträgliche Anpassen der VMs, Größe von Massenspeicher und RAM, ist möglich.

Für diese beiden Kategorien kann man wieder zwischen Servicekategorie Bronze und Silber auswählen. Die Servicekategorie Bronze wird für produktive Dienste mit geringen bis normalen Verfügbarkeitsansprüchen und für Testzwecke empfohlen, während die Servicekategorie Silber für hohe Verfügbarkeit, beispielsweise DARIAH Hosting Services eingesetzt wird.

Eine virtuelle Maschine wird standardmäßig den Nutzern inklusive Betriebssystem zur Verfügung gestellt. Verschiedene Betriebssysteme, auch SLES, Ubuntu LTS (Ubuntu mit Long-Term-Support) werden angeboten, und Windows-Betriebssysteme können genutzt werden. Allerdings muss die Lizenz, wenn benötigt, von dem Nutzer selbst beschafft werden.

Zusätzlich werden VMs mit Backup angeboten. Hierfür wird Tivoli Storage Manager, TSM, eingesetzt. Das gesamte Dateisystem der VM wird täglich gesichert und die Historie wird für 3 Monate aufbewahrt.

Außerdem werden VMs auch nach Wunsch mit Betriebssystemwartung angeboten. Eine Wartung des Betriebssystems umfasst unter anderem die Aktualisierung von installierten Paketen, Einspielen von Sicherheits-Patches, Installation von Software. Bei LTS-Versionen eines Betriebssystems kann innerhalb des Hersteller-Unterstützungszeitraums das System in derselben Version verbleiben.

Diverse technische Beratung und Unterstützung werden angeboten. Beispiele hierfür sind:

Technische Beratung und Unterstützung von Anfragen (A.2), Konfiguration und Installation eines DARIAH Service (A.3), Wartung (A.4). Auf Wunsch der Nutzer wird ein existierender Service angepasst, erweitert oder neu implementiert. Ein Beispiel ist die Implementation des Portal Services, dieser wird anhand von LifeRay auf Wunsch entwickelt (A.5 Implementierung). Zusätzlich zur Bereitstellung von virtuellen Maschinen mit Betriebssystem, Backup und Betriebssystemwartung kann auch die Wartung der Applikationssoftware vereinbart werden (A.6 Hosting von Komplett-Services).

\subsection{Services Basisdienste}

\subsubsection{PID-Service}

PID- und PID-Resolution, Persistent Identifier, bietet einen eindeutigen und persistenten Identifizierer für digitale $\mathrm{Ob}$ jekte, beispielsweise digitale Publikationen, Images oder Videos. Es wird ein zuverlässiger Zugriff über lange Zeiträume auf die digitalen Objekte gewährleistet. Zurzeit wird der PID-Service auf der Basis der hochverfügbaren ePIC²Infrastruktur, angeboten. Aktuell werden zwei Serviceklassen des PID-Services angeboten (B.1 PID-Service):

(a) maximal 50000 PIDs oder

(b) maximal 1 Million PIDs.

Sollte dies nicht ausreichen, können zusätzlich angepasste PID-Services angeboten werden.

2 Siehe www.pidconsortium.eu und Kálmán, Kurzawe und Schwardmann (2012). 


\subsubsection{Storage}

DARIAH-DE Storage Service bietet Geisteswissenschaftlern diverse kundengerechte Speicher-Services, die ihre Daten verlässlich und nachhaltig aufbewahren und gleichzeitig ihre unterschiedlichen Bedürfnisse befriedigen (B.2 Storage).

Es werden unterschiedliche Dateisysteme zur Speicherung von Daten den Nutzern angeboten. Diese Systeme sind zur Erhöhung der Ausfallsicherheit redundant ausgelegt. Eine tägliche Datensicherung, Backup, wird durchgeführt (B.2.1 Fileserver/Massenspeicher).

Die Daten, die selten oder viel später wieder genutzt werden sollen, zum Beispiel Dateien eines abgeschlossenen Projektes oder Datensammlungen, können auf kostengünstigen Speichern dauerhaft ausgelagert werden. Hierfür wird ein Hierachical Storage System, HSM, das sich transparent in das Dateisystem einfügt, bereitgestellt. Es umfasst einen Plattenspeicher und ein Kassetten-Robotsystem. Der Plattenspeicher dient als Zwischenablage archivierter Daten. Ins Archiv verschobene Daten werden zunächst im Cache gespeichert und später automatisch an zwei redundanten Standorten auf Kassetten kopiert (B.2.2 Langfristige Datensicherung).

Das DARIAH-Repositorium ermöglicht es Nutzern, ihre digitalen Objekte einfach, sicher und nachhaltig $\mathrm{zu}$ archivieren. Dies ist sowohl über eine Web-Schnittstelle per Browser als auch über eine API zugreifbar, und so können die digitalen Objekte der Fachcommunity langfristig zur Verfügung gestellt werden. Die Daten werden zunächst mit DC-Metadaten, Dublin Core Metadata, ${ }^{3}$ ausgezeichnet. Nach dem Einspielen der Daten sind sie anhand der PID referenzierbar und mithilfe der DARIAH generischen Suche recherchierbar, und somit öffentlich zugänglich (B.2.3 DARIAH-Repositorium (Data Curation)).

DARIAH Storage Service, ${ }^{4}$ ist ein standardisierter, auf REST $^{5}$ und HTTP ${ }^{6}$ basierender Service (B.2.4 DARIAH Storage Service (Bitstream Preservation)). Und er ist auch die Basisschnittstelle für höherwertige Dienste wie DARIAHRepositorium. Bitstream Preservation bietet die grundlegende Archivierung digitaler Daten, bei der die physischen Datenobjekte erhalten und somit die digitalen Daten langfristig verfügbar gehalten werden. Der physische Erhalt der Datenobjekte, Bitstreams, wird nach Wunsch der Kunden auf entsprechenden Speichermedien, beispielsweise Festplatten oder Magnetbändern, gesichert. Die Da-

$3 \mathrm{http} / / /$ dublincore.org.

4 Vgl. Tonne et al. (2013) und Schmitt et al. (2014).

5 http://www.ics.uci.edu/ fielding/pubs/dissertation/top.htm.

6 http://www.w3.org/Protocols. tenintegrität wird durch Bit-Preservation-Maßnahmen innerhalb des Services gewährleistet. Einzelne Dateien können hochgeladen, aktualisiert, heruntergeladen oder gelöscht werden. Unabhängig von den Datenformaten, Datenmengen und Inhalten, können heterogene Daten, gleichmäßig behandelt werden. Eine webbasierte Schnittstelle zum Manipulieren der Daten, wird angeboten. AAI garantiert dabei einen abgesicherten Zugang zum System. Die folgende Operationen/Funktionen stehen für die digitalen Daten zur Verfügung:

- Erstellen/hochladen (create),

- Lesen/herunterladen (read),

- Ändern/aktualisieren (update),

- Löschen (delete).

Mit dem Cloud Share Service können Nutzer-Daten überall auf dem gleichen Stand gehalten (synchronisiert) werden. Dies wird ermöglicht durch auf PCs und Laptops, aber auch auf Handys und Tablets installierten Client-Programmen. Die Daten können auf einen Server per Browser gespeichert und dann mit anderen geteilt oder auf andere Geräte übertragen werden. Man kann auch offline an den Dateien arbeiten, Änderungen werden dann bei der nächsten Online-Verbindung übertragen und empfangen (B.2.5 Cloud Share (DFN-Cloud)).

Es werden für unterschiedliche Einsatzbereiche der Nutzer Datenbankserver angeboten. Unter anderem wird ein Server für MySQL-Anwendungen angeboten. Dabei wird eine schnelle und hochverfügbare Anbindung zum MySQL-Dienst sichergestellt. Der verwendete Server ist speziell für MySQL-Anwendungen ausgelegt und redundant vorhanden. Durch permanente Replikation der Daten ist der Zugriff auf den jeweils aktuellen Datenbestand sichergestellt (B.3 Datenbanken).

\subsubsection{Kollaborative Arbeitsumgebungen}

Geistes- und Kulturwissenschaften, wie jede andere wissenschaftliche Disziplin, entfaltet zunehmend Bedarf an computergestützten kollaborativen Arbeiten. DARIAH-DE bietet hierfür diverse Services, bei denen Werkzeuge und Tools einer digitalen Forschungsinfrastruktur für die kollaborativen Arbeitsumgebungen realisiert werden.

Die Nutzung von Mailinglisten ermöglicht eine einfache E-Mail-Kommunikation unter den gleichgesinnten Wissenschaftlern. Eine Mailingliste erlaubt eine beliebige Anzahl von E-Mail-Adressen einzutragen. Wird eine E-Mail an eine Mailingliste versendet, wird sie automatisch an alle eingetragenen E-Mail-Adressen der Mailingliste verteilt. Administratoren von Mailinglisten können E-Mail-Adres- 
sen in ihre Liste eintragen und wieder löschen. Die Parameter ihrer Liste können eingestellt und Informations- und Willkommensnachrichten erstellt werden. Für die Mailinglisten wird Mailman eingesetzt (B.4 Service Mailinglisten).

Ein webbasierter Service, basierend auf LimeSurvey, für die Erstellung von Umfragen wird angeboten. Die Umfragen werden auf einfache Weise im Webbrowser mit Mausklick erstellt, welcher keine Programmierkenntnisse erfordert. Vielfältige Möglichkeiten zur Gestaltung von unterschiedlichen Typen von Umfragen werden angeboten. Der Survey-Benutzer kann die eigenen Umfragen und erhobenen Daten exportieren und ist verantwortlich für ihre sichere Lagerung. Die Umfragedaten, die älter als 6 Monate sind, werden standardmäßig nach Abschluss einer Umfrage von ihren Servern gelöscht (B.5 Technical Survey Provisioning).

Ein Enterprise-Wiki-Service wird angeboten, der für die Kommunikation, Dokumentation und den Wissensaustausch flexibel verwendet werden kann (B.6 Confluence).

Der Web-basierte Editor, EtherPad, zum kollaborativen Bearbeiten von Texten wird bereitgestellt. Mit EtherPad können mehrere Personen in Echtzeit unabhängig von den Standorten und Softwareausstattungen gemeinsam an dem gleichen Text arbeiten. Somit wird das kollaborative Schreiben wesentlich erleichtert (B.7 EtherPad).

Auch kann der schon genannte Cloud Share Service zur gemeinsamen Arbeit an Dokumenten und Dateien genutzt werden, indem Gruppen von Nutzern der Zugang zur Synchronisation von Verzeichnissen erlaubt wird.

\subsubsection{Developer Portal}

In Kombination mit den kollaborativen Arbeitsumgebungen wird das Developer Portal ${ }^{7}$ für die Realisierung der Digital Humanities (DH) Forschungs- und Entwicklungsprojekte bereitgestellt.

Es wird ein Web-basiertes kollaboratives Projektmanagementsystem bereitgestellt, das den kompletten Projekt-Lebenszyklus unterstützt, unter anderem das Erstellen und die Diskussion von Projektplänen, Issue Tracking, Fortschrittsberichte und Austausch von Wissen (B.8 Projektverwaltung ${ }^{8}$ ). Es beinhaltet Version-Control-Systeme wie GIT und SVN, die für eine kontrollierte gemeinsame Softwareentwicklung eingesetzt werden können.

Eine Anwendung, die kontinuierliche Integration und kontinuierliche Lieferung von Software unterstützt und somit hilft, die Produktivität der Entwicklungsteams zu steigern, wird mit Jenkins angeboten. Hiermit können Software-Projekte kontinuierlich kompiliert und getestet werden, was die Integration von Änderungen für Entwickler und das Erstellen neuer Releases für die Benutzer deutlich erleichtert. Jenkins bietet eine Reihe von Plugins, die unterschiedliche Anforderungen und Nutzungsszenarien der DH-Developer-Community abdecken (B.9 Jenkins).

\subsection{Services Generische Dienste}

Methoden, Verfahren und Tools der Digital Humanities bieten neue Möglichkeiten für die Forschung. Generische Dienste unterstützen hierfür digital arbeitende Geistesund Kulturwissenschaftler unabhängig von der speziellen Forschungsdisziplin in ihrem Forschungsprozess. DARIAH-DE bietet auch die Entwicklung von generischen Diensten auf Anforderung an.

Ein Beispiel ist der DARIAH-DE-Geo-Browser, der eine Softwarelösung für die webbasiertes Visualisierung von Raum-Zeit-Relationen von Daten bereitstellt.

Mehrere Anfragen können dabei miteinander verglichen werden (C.1 GeoBrowser ${ }^{9}$ ).

Die Collection Registry ist ein webbasierter Service, der Informationen über Forschungsdatensammlungen wie Bücher, Bilder oder Statuen, vereint und den Nutzern zur Verfügung stellt. Es werden allgemeine Informationen über Sammlungen, eine Sammlungsbeschreibung, wie Standort und Zugriffspunkte auf die Sammlung sowie Metadaten wie die räumliche oder zeitliche Spanne der Sammlungselemente und ihrer Inhalte verzeichnet. Die Collection Registry ist durchsuchbar und Nutzer können eine neue Sammlungsbeschreibung anlegen oder Bestehende ändern. Ein sicherer Zugang zur Collection Registry wird durch AAI garantiert (C.2 Collection Registry ${ }^{10}$ ).

Die Schema Registry bietet konzeptionelle Hilfe bei der Strukturierung nicht standardisierter (Meta-)Daten oder der Abbildung zwischen Metadaten mit verschiedenen Standards (Mapping). Es werden semi-strukturierte Datenmodelle und Korrelationen zwischen ihnen beschrieben. Die Spezifikation von Strukturen z. B. im XML-Schema können in Bezug auf eine Kollektion erweitert werden, die Semantik originärer Daten bleibt erhalten (C.3 Schema Registry).

Die Generische Suche bietet eine übergreifende Suchmöglichkeit. Dafür werden die einzelnen Metadatensätze

9 https://de.dariah.eu/geobrowser, http://geobrowser.de.dariah.eu.

$10 \mathrm{http}: / /$ colreg.de.dariah.eu.
7 https://de.dariah.eu/developer-portal.

8 https://projects.gwdg.de. 


\section{Übersicht über die Infrastruktur}

Digitale Forschungsinfrastruktur für die

Geistes-und Kulturwissenschaften

Rechenzentren

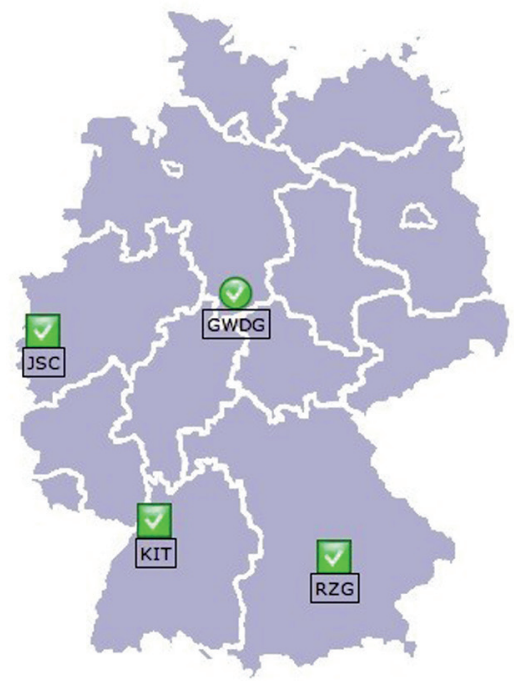

Dienste

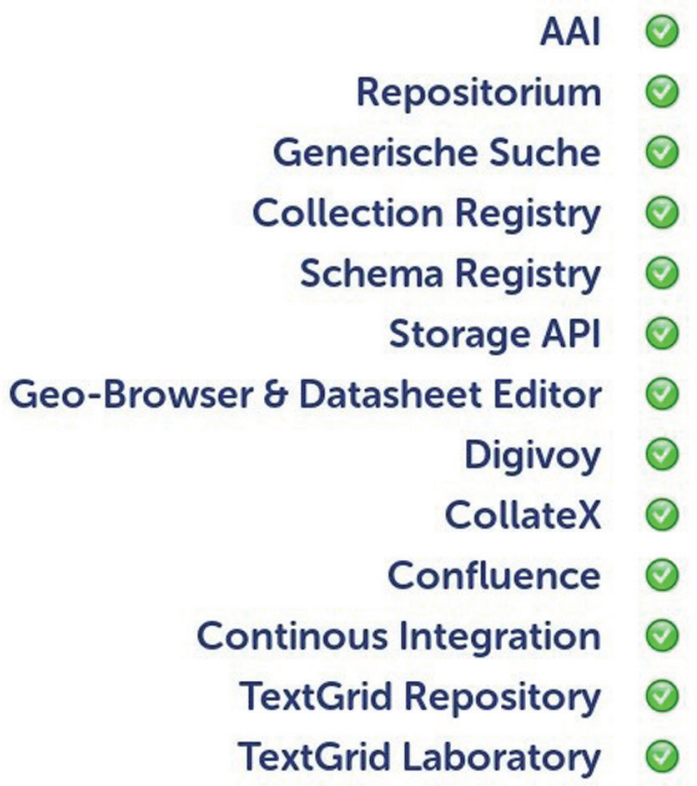

Abb. 4: Monitoring

der in der Collection Registry beschriebenen Sammlungen zusammengetragen. Es ist möglich, sich eine eigene Auswahl der Sammlungen zusammenzustellen und dauerhaft zu speichern, und dies mit anderen Nutzern zu teilen. Die Generische Suche vereint die Eigenschaften von Breitenund Tiefensuche unter Anwendung der in der DARIAH-DE Crosswalk Registry definierten Assoziationen und Transformationsregeln und ermöglicht eine dynamische Anpassung der Suche (C.4 Generische Suche ${ }^{11}$ ).

\subsection{Service Operative IT-Dienste}

Die operativen IT-Dienste sind ein essentieller Baustein jeder produktiven Forschungsinfrastruktur. Im Fall von DARIAH-DE werden hier Softwarekomponente und Werkzeuge von hoher Qualität bereitgehalten, die für den reibungslosen täglichen Betrieb sorgen. Im Folgenden wird auf die drei wichtigsten operativen Dienste eingegangen.

11 http://search.de.dariah.eu/search.

\subsubsection{Monitoring von Diensten und Werkzeugen}

Die Überwachung von Infrastrukturkomponenten und Diensten, also das Monitoring, ist für den täglichen Betrieb einer Forschungsinfrastruktur unerlässlich. Das DARIAHDE-Monitoring ermöglicht die Abfrage des allgemeinen $\mathrm{Zu}$ standes der Infrastrukturkomponenten, die Sicherstellung der Erreichbarkeit der Systeme sowie die allgemeine Überwachung der verteilten Dienste und Server. Dadurch ist es möglich, auftretende Probleme zu identifizieren und Betriebsstörungen schnellstmöglich, teilweise automatisch zu beheben. Bei jedem Ereignis werden auch die verantwortlichen Personen benachrichtigt, um gegebenenfalls ein schnelles manuelles Eingreifen zu ermöglichen.

Das DARIAH-DE-Monitoring wurde so konzipiert, dass es sowohl die Administratoren, als auch die Nutzer der Forschungsinfrastruktur unterstützt, indem verschiedene Sichten generiert und die Vielfältigkeit der bereitgestellten Dienste berücksichtigt werden (vgl. Abb. 4).

Außerdem ist es vorgesehen, die Einhaltung von Dienstgütevereinbarungen (SLA) mithilfe dieses Monitoringsystems zu überwachen. 


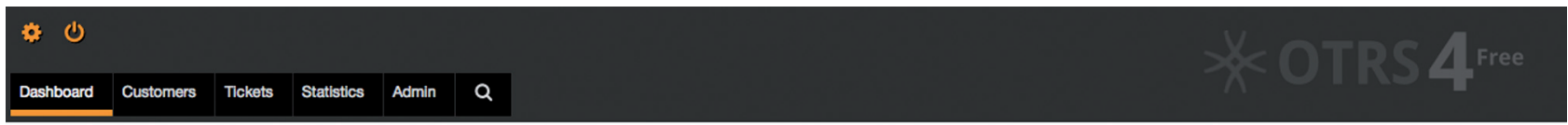

\section{Dashboard}
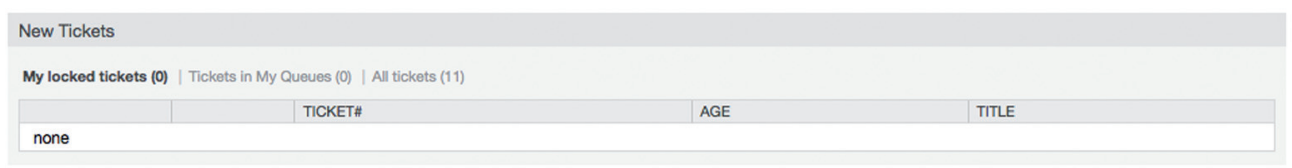

Open Tickets / Need to be answered

My locked tickets (0) | Tickets in My Queues (0) | All tickets (23)

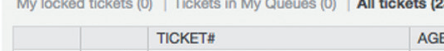

\begin{tabular}{|c|c|c|c|}
\hline & & & \\
\hline$\square$ & 23 & 2016021042000021 & $19 \mathrm{~h} 25 \mathrm{~m}$ \\
\hline 믄 & & 2016020942000014 & $1 \mathrm{~d} 21 \mathrm{~h}$ \\
\hline$\square$ & $\omega$ & 2016020542000012 & $6 \mathrm{~d} 6 \mathrm{~h}$ \\
\hline$\square$ & $\hat{\imath}$ & 2016020442000023 & $6 \mathrm{~d} 22 \mathrm{~h}$ \\
\hline - & का & 2016020242000027 & $9 \mathrm{~d} 2 \mathrm{~h}$ \\
\hline$\square$ & $\hat{\omega}$ & 2016013142000019 & $11 \mathrm{~d} 0 \mathrm{~h}$ \\
\hline$=$ & 它 & 2016012542000031 & $17 \mathrm{~d} 3 \mathrm{~h}$ \\
\hline$\square$ & 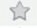 & 2016012542000021 & $17 \mathrm{~d} 3 \mathrm{~h}$ \\
\hline 믄 & 定 & 2016012542000012 & $17 \mathrm{~d} 3 \mathrm{~h}$ \\
\hline ㅁ & $\hat{\imath}$ & 2016012242000072 & $19 \mathrm{~d} 23 \mathrm{~h}$ \\
\hline
\end{tabular}

$$
\text { TITLE }
$$

[DARIAH register@dariah.eu] Accountanfrage von textgrid.de

Zugang Confluence-Space

request for approval

FW: Humanities at Scale - WP 6

access request to HaS-Dariah wiki

[DARIAH registeradariah.eu] Accountanfrage von textgrid.de

[DARIAH register@dariah.eu] Starleaf Account

[DARIAH register@dariah.eu] HaS Confluence account

[DARIAH register@dariah.eu] Starleaf Account

[DARIAH register@dariah.eu] Accountanfrage von textgrid.de

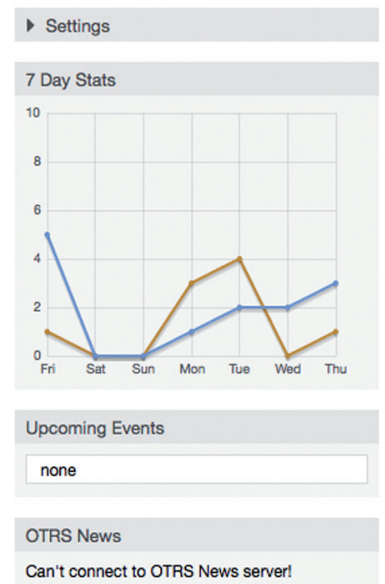

Ticket Queue Overview

QUEUE

Nicht zugeordnet

TOTALS

NEW
0

0

0

OPEN

2

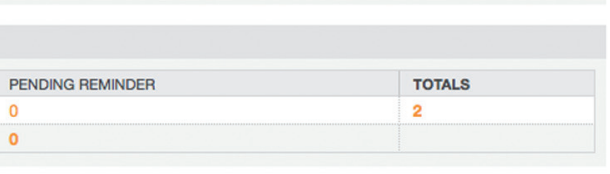

0

TOTALS

Can't connect to OTRS News server!

Abb. 5: Helpdesk

\subsubsection{Helpdesk und zentrale Unterstützung}

Im Fall der DARIAH-DE-Forschungsinfrastruktur unterstützt ein Helpdesk oder Supportsystem sowohl die Bearbeitung von Anfragen als auch den Aufbau einer Wissensdatenbank. Für Letztere wird der gesamte Verlauf im Supportsystem inklusive aller Antworten hinterlegt. Somit werden die behandelten Themenfelder, die vielfältigen Fragestellungen sowie die Stellungnahmen der fachwissenschaftlichen und technischen Experten dokumentiert.

Für die Bearbeitung von Anfragen stellt das System sicher, dass ein Gesamtüberblick über die zu bearbeitenden Vorgänge und die Gesamtkommunikation zu jeder Zeit möglich ist. Die Aufgaben und Fragestellungen werden in sogenannten Tickets zusammengefasst. Das Helpdesksystem von DARIAH-DE hilft beim Empfang, bei der Klassifizierung, bei der Bestätigung und bei der Bearbeitung von Anfragen. Die Zuweisung zu einer Person oder Personengruppe kann auch automatisch erfolgen, was besonders die generischen Abläufe einer Forschungsinfrastruktur sehr gut unterstützen kann (siehe Abb. 5).

\subsubsection{Authentifizierungs- und Autorisierungsinfrastruk- tur (AAI)}

Die Komponente der DARIAH-Authentifizierungs- und Autorisierungsinfrastruktur (DARIAH AAI) stellt sicher, dass Anfragen von Nutzern wirklich von ihnen stammen (Authentifizierung) und dass sie die Berechtigung für die Nutzung einer bestimmten Ressource haben (Autorisierung). Die Unterstützung von Standards und die Verwendung von standardisierten Schnittstellen sind auch im Bereich AAI sehr wichtig, um ein gemeinsames Vokabular zwischen den teilnehmenden Einrichtungen und der Forschungsinfrastruktur zu ermöglichen. Die Komponente der DARIAH AAI basieren auf internationalen Standards, die sich sowohl in Hochschulbereich als auch in der Industrie haben durchsetzen können. So können die Forscher aus Verbünden akademischer Einrichtungen, aber auch interessierte Laien ohne Zugehörigkeit $\mathrm{zu}$ einer Forschungseinrichtung die Dienste und Ressourcen der Forschungsinfrastruktur gleichzeitig mitnutzen. 


\section{Einbindung fachspezifischer Infrastrukturkomponenten}

Die nachhaltige Einbindung von fachspezifischen Infrastrukturkomponenten in einer Forschungsinfrastruktur wie DARIAH-DE ist ein komplexer Prozess. Während dieses Prozesses muss man neben den fachwissenschaftlichen auch die technischen Anforderungen berücksichtigen. Dieser Dialog wird im Fall von DARIAH-DE von fachwissenschaftlichen und technischen Mentoren begleitet. Für den kompletten Integrationsprozess sind 5 Phasen definiert (siehe Abb. 6).

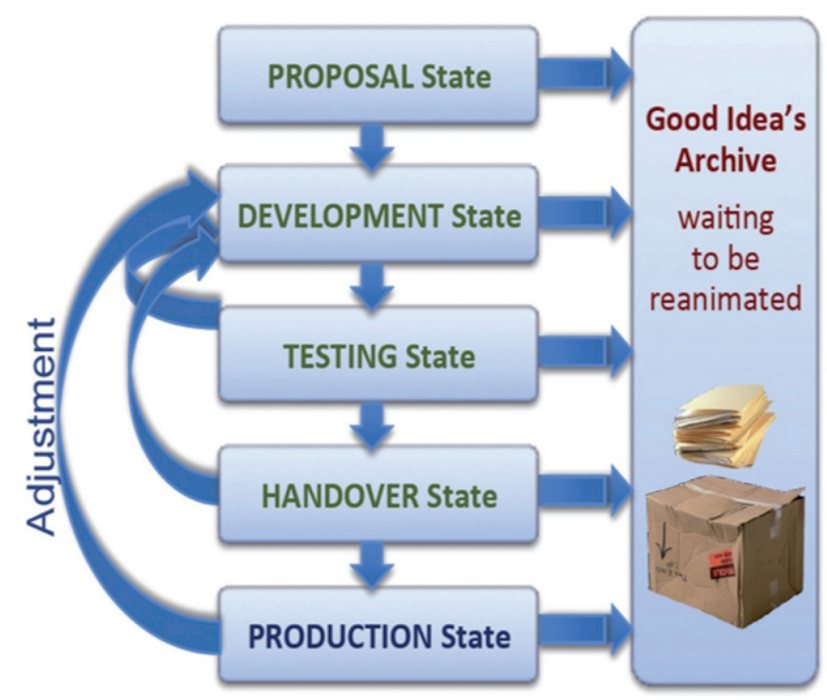

Abb. 6: Service Life Cycle von der DARIAH-DE Forschungsinfrastruktur $^{12}$

In der ersten Phase, dem Proposal State, werden Mentoren benannt und es wird evaluiert, ob die neue Komponente oder das Werkzeug sich in die DARIAH-Forschungsinfrastruktur integrieren lässt. Nach einer Entscheidung folgen die Weiterentwicklungen der Komponente im Development State. Hier wird auch deren Dokumentation weiterentwickelt und die Komponente wird in die DARIAH-Infrastruktur eingebunden. Um den DARIAH-Ansprüchen gerecht zu werden, wird der Dienst oder das Werkzeug in dem Testing State von geistes- und kulturwissenschaftlichen Nutzergruppen getestet und deren Rückmeldung eingeholt. Bei Bedarf wird die Komponente weiterentwickelt. Im Anschluss an die Entwicklung und das Testen werden die Software, Dokumentation, Daten usw. einem Service
Hosting Team übergeben. Die letzte Phase, der Production State, schließt den komplexen Vorgang der Integration in einer Forschungsinfrastruktur ab. Hier wird der Dienst oder das Werkzeug von DARIAH-DE nachhaltig gepflegt und allen Benutzern zur Verfügung gestellt.

\section{Innovationszyklen und Nachhaltigkeit der Angebote am Beispiel von DARIAH-DE}

Infrastrukturkomponenten, Dienste und Werkzeuge unterliegen rasanten Entwicklungen. Im Rahmen von Forschungsprojekten entstehende oder während kürzerer Förderzeiträumen von Zeit zu Zeit angepasste Werkzeuge können nach dem Auslauf der Finanzierung oft nicht mehr koordiniert gepflegt oder weiterentwickelt werden. Aber nur wenn Infrastrukturkomponente und Werkzeuge längerfristig erhalten bleiben, wird Vertrauen in eine Forschungsinfrastruktur wie DARIAH-DE entstehen. Daher spielt die Nachhaltigkeit von Komponenten und Nachnutzung von Angeboten in DARIAH eine wichtige Rolle. Sie lässt sich in verschiedene Teilaspekte unterteilen: betriebliche, fachwissenschaftliche, datentechnische, organisatorische und technische Nachhaltigkeit. Im Folgenden werden die technische und die datentechnische Nachhaltigkeit beschrieben.

Bei den technischen Basisdiensten soll zum einen der modularen Ansatz, aufbauend auf generischen technischen Komponenten, gewählt werden, zum anderen sollen Standards und standardisierten Schnittstellen verwendet werden. Technische Komponenten, aber auch Technologien unterliegen einem ständigen Wandel, was in regelmäßigen Abständen zu ihrer Erneuerung führt. Die Verwendung von generischen Komponenten und standardisierten Schnittstellen erleichtern diesen Prozess und garantieren der Gesamtinfrastruktur eine größere Stabilität.

Der Zugang zu Daten, die technische Interoperabilität von Daten sowie die Interoperabilität von Datenverarbeitungswerkzeuge fallen in den Bereich von datentechnischer Nachhaltigkeit. Im Fall der DARIAH-DEForschungsinfrastruktur werden diese Fragestellungen insbesondere durch konzeptuelle und organisatorische Maßnahmen adressiert. So werden Empfehlungen zu Prozeduren, Organisationsstrukturen und generische Standards ausgearbeitet. 


\section{Zusammenfassung}

Seitdem mithilfe von Informationstechnologie Dienste angeboten werden, sind diese jeweils rasanten Entwicklungen unterlegen, was zu immer neuen Möglichkeiten, entsprechend auch wachsenden Anforderungen und vielfach $\mathrm{zu}$ einer hohen Unübersichtlichkeit des Angebotes und Komplexität der Lösungen geführt hat. Der modulare Ansatz, aufbauend auf generischen technischen Basiskomponenten zusammen mit einem organisatorischen Konzept der Anpassung an neue technische Möglichkeiten und neue wissenschaftliche Anforderungen versucht in einer solchen Landschaft der Veränderung eine Balance zwischen Kundenfreundlichkeit und technischer Komplexität, zwischen Erhalt von erprobten Diensten und technischen Innovationen herzustellen.

Dies ermöglicht den steten Austausch mit den Anforderungen der Wissenschaftler und eine anschließende Anpassung der Dienste an sich neu entwickelnde Technologien wie auch wissenschaftliche Fragestellungen. Gleichzeitig können die Wissenschaftler bei ihrer Arbeit von teilweise stark spezialisierten Diensten eine ähnliche Dienstgüte erwarten, wie sie sie bei der Nutzung von EMail oder sozialen Netzen gewöhnt sind.

\section{Literaturverzeichnis}

Kálmán, Tibor; Kurzawe, Daniel; Schwardmann, Ulrich (2012): European Persistent Identifier Consortium - PIDs für die Wissenschaft. In: Altenhöner, Reinhard; Oellers, Claudia (Hrsg.): Langzeitarchivierung von Forschungsdaten - Standards und disziplinspezifische Lösungen. Berlin: Scivero, 151-168.

Tonne, Danah; Rybicki, Jedrzej; Funk, Stefan E.; Gietz, Peter (2013): Access to the DARIAH Bit Preservation Service for Humanities Research Data. In: $21^{\text {st }}$ Euromicro International Conference on Parallel, Distributed and Network-Based Processing (PDP 2013). Belfast, 9-15.
Schmitt, Oliver; Siemon, Andreas; Schwardmann, Ulrich; Hellkamp, Marcel (2014): GWDG Object Storage and Search Solution for Research Common Data Storage Architecture (CDSTAR). (GWDG Bericht, 78). Göttingen: GWDG. Verfügbar unter https://www.gw dg.de/documents/20182/31188/gwdg-bericht-78.pdf/4e2436 a7-1054-4cc5-9f5d-8af4baaeb51f.

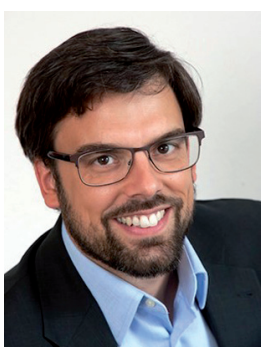

Tibor Kálmán

Gesellschaft für wissenschaftliche Datenverarbeitung mbH Göttingen Arbeitsgruppe eScience Am Fassberg 11 D-37077 Göttingen tkalman@gwdg.de

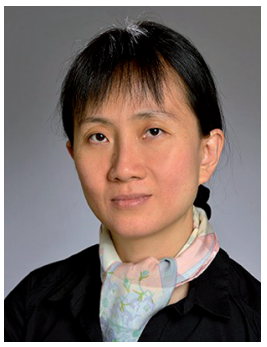

\section{Xi Kong}

Gesellschaft für wissenschaftliche Datenverarbeitung mbH Göttingen Arbeitsgruppe eScience Am Fassberg 11 D-37077 Göttingen xi.kong@gwdg.de

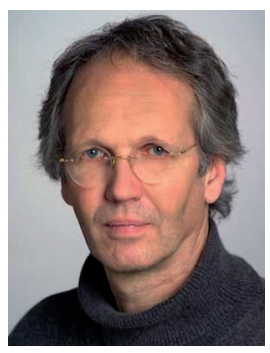

Ulrich Schwardmann

Gesellschaft für wissenschaftliche Datenverarbeitung mbH Göttingen Arbeitsgruppe eScience Am Fassberg 11 D-37077 Göttingen uschwar1@gwdg.de 